\title{
Exploring the Comparative Efficacies of Allopathic and Homeopathic Treatments in Reducing Dermal Malassezia Folliculitis
}

\author{
Chelsea Borja ${ }^{1}$ and Russell Aaronson\# \\ ${ }^{1}$ Coral Springs High School, Coral Springs, FL, USA \\ \#Advisor
}

\section{ABSTRACT}

Malassezia folliculitis is a condition in which the naturally occurring yeasts on the skin proliferate within the sebaceous glands and cause inflammation. The lipophilic Malassezia yeasts colonize the sebaceous glands of the skin and wreak havoc on the skin, leaving patients and dermatologists alike, unhappy. The issue is further exacerbated by a lack of research on the comparative efficacies of treatment types alternative to those heavily implemented in the public sphere, such as allopathic and homeopathic medicines. This paper seeks to narrow this knowledge gap and identify potentially more efficacious treatments to eradicate MF than those already recognized by the public domain. Thus, such will be executed through a two-part, quantitative correlational study that investigates response to both conventional and alternative treatment types to determine the most efficacious type of treatment for eradicating or reducing MF. To encompass both a personal and professional perspective, a questionnaire was distributed to patients who have had or currently have MF (the Patient Survey) and dermatologists who have treated or are currently treating patients with MF (the Dermatologist Survey). The data produced from both surveys (though more heavily supported by the Patient Survey as there were more responses) ultimately suggested that a combination treatment may be most ideal- a routine that incorporates both allopathic and homeopathic treatments taken both orally and topically.

\section{Introduction}

For centuries, yeasts of the genus Malassezia Folliculitis (MF) have colonized the skin of many. Malassezia (Pityrosporum) folliculitis is an inflammatory skin condition caused by an infection of the sebaceous glands with Malassezia [1]. The Malassezia yeasts are lipophilic, meaning that they are attracted to lipids, many of which conventionally manifest as oils, fats, esters, and waxes. When MF colonizes the sebaceous glands of the skin, which are glands that produce oil located in the dermal layer, it yields several skin-related complications such as irritation and inflammation, yeast-related breakouts, itching and burning. The yeast typically colonizes the face, back, extensor surfaces of the arms, chest, and neck [2]. Unlike typical complications of the acne vulgaris strain, MF does not respond to most antiacne treatments because of its lipid-based pathogenesis and proliferation that make it vehemently stubborn and unique to treat. This stubborn persistence on the skin and resistance to conventional acne treatments encourages exploration for newer ways to manage and treat the yeast overgrowth; thus, treatment for Malassezia, in terms of this paper, means: allopathic (synthetic, lab-designed), and homeopathic (naturally derived, organic) medicines, of which can be taken topically (applied externally to the skin) or orally (ingested through mouth).

A study included in the journal of Ethnopharmacology by Donato et al. reviewed published efficacies of essential oils as treatments for MF, which are a form of homeopathic treatment. The study concluded that essential oils as homeopathic medicine can prove worthy prospects for clinical application [5]. The study presents an important, foundational perspective for this paper, as it offers a perspective in favor of homeopathic treatment, a contrasting point of view. Moreover, studies have also found that oral medicine may prove more efficacious than topical medicine in 
reducing MF on the skin due to its ability to penetrate the hair follicles from deep within [1]. For example, two common medicines for MF have been identified as Ketoconazole and Itraconazole, both of which can be orally ingested or applied topically. It is evident that there are several different available treatment options for Malassezia that, too, can be ingested orally or applied topically, aside from Ketoconazole and Itraconazole.

Even so, however, the consensus is that the ultimate solution to reducing MF most effectively remains unclear, a randomized correlational study by Abderrahmen et al., notes [3]. MF often wreaks havoc on the skin, causing the aforementioned uncomfortable reactions, leaving patients and dermatologists alike, distraught. Thus, this paper seeks to determine the type of treatment most efficacious in reducing the presence and proliferation of dermal Malassezia Folliculitis through a correlational research study that investigates response to alternate treatment types.

\section{Review of Literature}

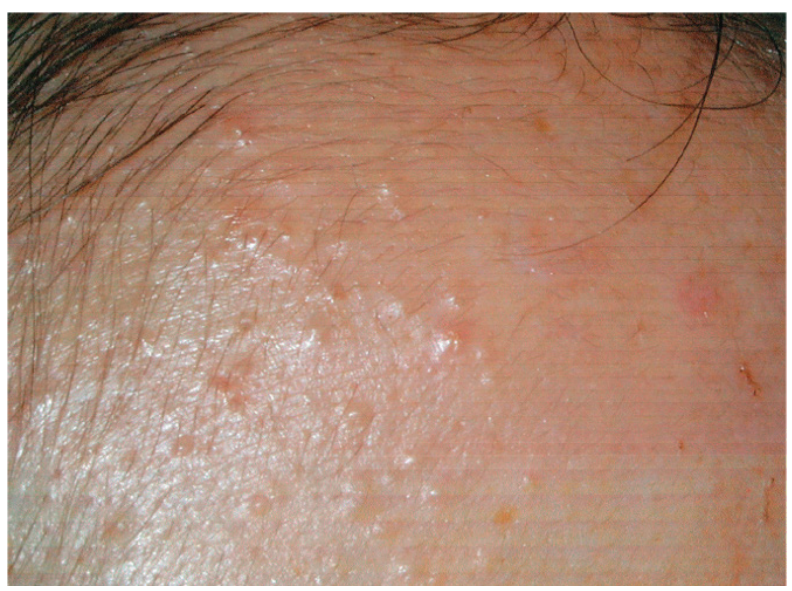

Figure 1. Close up view of patient showing multiple Malassezia pustules; Sweeney et al., 2005. [4].

To preface, it is important to differentiate between the circulating definitions surrounding Malassezia Folliculitis. Though commonly referred to as Pityrosporum Folliculitis, the term does not appear to live up to the status quo in dermatology. In some research, it is described as a common inflammatory skin disorder that may mimic acne vulgaris [4]. Pityrosporum was a previously coined term which prompted many dermatologists to consider 'Malassezia Folliculitis' a more modernly encompassing and fitting name for the condition. This is not to say that Pityrosporum is completely disregarded now, but rather that it is growing more uncommon in the sphere of dermatological research. Many researchers describe Malassezia Folliculitis as an inflammatory condition caused by infection with Malassezia yeast of the sebaceous glands [1]. Essentially, folliculitis is the inflammation or the reaction that occurs as a result of the overgrowth and colonization of the Malassezia yeast that is naturally present on the skin. For the purpose of this paper, the latter definition will be used instead because the broadness and ambiguity of the former definition fails to accurately encompass present-day dermatological findings.

Several factors have been recognized to interfere with Malassezia yeast pathogenesis: environment, climate, diet, and treatment type. A comparative study noted that predisposing factors of MF in their patients primarily included seborrhea (excessive oil production), excessive exercise, and excessive sweating. Furthermore, the study noted that the differences in yeast colonization on different areas of the body were likely due to these varying predisposing factors that would not be the same for everyone, resulting in different breakout areas [3]. Another group of researchers recorded that Malassezia folliculitis is more frequent in tropical parts of the world, likely because of the humidity and warm climate. The study went on to show that it is common among immunosuppressed patients, diabetics, as well as in patients in broad-spectrum antibiotic treatment [8]. As for factors that directly encourage proliferation, one study noted that antibiotics may alter normal skin flora and lead to the yeast's overgrowth [9]. Similarly, it showed that 
occlusive clothing and topical (externally applicable) products, such as make-up, lotion, or sunscreens can contribute to the proliferation of the Malassezia yeast and promote flares.

Moreover, the stubborn and complex nature of the yeast Malassezia prompts the existence of two dominating perspectives: those who assert that allopathic medicine is most effective in reducing the yeast and those who assert that homeopathic medicine is most effective. Within these perspectives, however, lie intricacies regarding the administering of medicine that will be addressed in further data collection if proved significant to the claim. For instance, it may be necessary to note if a medication were to be taken orally or applied topically if the findings demonstrate that a combination of both allopathic and naturopathic medicines administered topically and orally is most effective in reducing the Malassezia yeast. As some research has proposed, it is likely that a combination of medicine types would be best in hindering Malassezia. Here is an example regarding the administering of medicine (i.e., how it is taken): Systemic antifungal treatment is probably more effective than topical treatment, since it eliminates Malassezia located deeply within the hair follicles. Combined systemic and topical treatment may be favorable. [1] Reiterating, oral medicine may prove more efficacious than topical medicine in reducing MF on the skin due to its ability to penetrate the hair follicles from deep within [1]. The disagreements persist- In a study's 6-week follow-up, those who had received systemic ketoconazole, or the combination of systemic and topical treatment had a clearance rate of $75 \%$ and $100 \%$, compared to $9 \%$ in the topical treatment group [6]. Upon interpretation, the data reads that systemic (oral) medicine is superior in treating Malassezia folliculitis than topical medicine. Also, two common medicines for MF have been identified as Ketoconazole and Itraconazole, both of which, again, can be orally ingested or applied topically.

There is a similar dynamic with the efficacies of allopathic vs homeopathic medicines for treating Malassezia. A study that tested the efficacy of essential oils in reducing the Malassezia yeast concluded that essential oils are promising active treatments against Malassezia infections. It said further, that, in some cases, combinations of different essential oils present synergistic effects, meaning that they work efficiently together over time. Despite these findings, many perspectives seem to lean towards allopathic means. Further research found that topical antifungals are the most used therapy to treat Malassezia-related diseases and that azoles and polyenes (amphotericin B), are frequently employed to treat Malassezia-related skin disorders or infections in humans, with Itraconazole, Ketoconazole, and Posaconazole being the most effective drugs [7]. Thus, the debate that has amassed as a result of multiple studies and experiments unable to reach a consensus on the most efficacious treatment type presents a question: what is the extent to which the comparative efficacies of allopathic and homeopathic treatments affect the presence and proliferation of dermal Malassezia folliculitis?

The focus is not solely on how the medicine is taken, (though such may appear significant in the later data) but more solidly on the efficacy of treatment types (allopathic, homeopathic) in reducing MF. Reiterating, since most studies argue either in favor of topical or oral allopathic medicine, the lack of arguments in favor of homeopathic medicine becomes ever-increasingly more prominent. It becomes more apparent, also, that research neglects the exploration of the nature of homeopathic alternatives to allopathic, in general. Thus, this paper seeks to exceed those limitations and identify the most efficacious medicine type for treating Malassezia folliculitis, which will resultantly uncover the more profound and often neglected nature and behavior of homeopathic medicines.

\section{Methods}

This study seeks to determine the type of treatment most efficacious in reducing the presence and proliferation of dermal Malassezia Folliculitis through a correlational research study that investigates response to alternate treatment types. Since there are numerous treatment types [7] the findings that would potentially generate from this study are critical as they can assist the dermatological scene of treatment development and distribution, thus improving the overall well-being of the patients.

Originally, the most desired method of data collection was an in-person, doctor monitored experimental study in which treatment type and efficacy as it relates to MF pathology would be observed in a laboratory. Due to restrictions set by COVID-19, doctor's offices declined this approach. As a result, the types of treatment given and their 
efficacies were instead analyzed through two correlational survey questionnaires that investigated response to different treatment types for MF. The two sets of expressed data: Patient experience and Dermatologist experience, were used to construct the "Patient Survey" (Appendix A) and "Dermatologist Survey" (Appendix B) models that would outline the findings of this study. Both perspectives were relevant to the study as reports from dermatologists who have professionally dealt with MF would provide insight on the nuances of MF and its responses to generic medical treatments, likely allopathic ones, whereas reports from patients would likely generate data about treatment alternatives, such as homeopathic ones. Thus, the two categorical distinctions of the data served to contextualize the general trends in practices surrounding the treatment of MF. Both the parallels in data amongst the personal perspective of a patient and the professional perspective of a dermatologist intersect the potential efficacies of allopathic and homeopathic treatments alike in improving MF.

The selection process- though broader for the aims of this specific research- varied for both perspectives. The study employed "Convenience Sampling," or the data collection technique that holds that participants are selected by the researcher's convenience; in other words, open to whomever is interested. Within the context of this study, the survey questionnaire was open to whomever was 'available' to participate. Dermatologists were contacted in 3 ways: In local dermatology offices, via an email through Gmail that contained the link to the survey, in-person flyer distributions to dermatology offices containing the link for the survey, and through phone calls where the link to the survey was given. By contrast, patient respondents were found strictly on social media platforms, specifically Reddit and Facebook Groups. Prior to entering the questionnaire section of the survey, both parties had to agree on their respective consent forms in order to participate. To ensure confidentiality, no personal information such as: names, organization, contact information, was asked of the participants. Information such as location; however, was asked, but was an optional, "open-ended" field in order to meet confidentiality guidelines. To eliminate any potential conflicts and ethical issues, the study design, methods, and procedures were approved by the Inquiry Review Board (IRB). After the agreement of consent, participants were able to move on to the rest of the survey, where the questionnaire began.

The survey contains set restrictions to ensure recipients meet the qualifications to participate: For the Patient Survey, participants must verify that they have been professionally diagnosed with MF before or have a current diagnosis at the time of participating in the survey. For the Dermatologist Survey, dermatologists must verify that they have treated MF before or are currently treating MF at the time of participating in the survey. Both parties must confirm their initial respective responses prior to entering the rest of the survey, as failure to do so will remove them from participating.

Both the "Patient Survey" and "Dermatologist Survey" questionnaires divided data into distinct sections relating to treatment type: allopathic or homeopathic treatment, and whether taken orally or applied topically. Under this, data was to be generated and classified under 9 treatment-related categories. For reference, the notations are of the following format: [Treatment type - Manner in which taken]. The 9 treatment-related categories were: [allopathictopical], [allopathic-oral], [allopathic- topical + oral], [homeopathic-topical], [homeopathic-oral], [homeopathic- topical + oral], [allopathic + homeopathic-topical], [allopathic + homeopathic-oral], [allopathic + homeopathic- topical + oral]. This organized data so that it would be more easily identifiable, classifiable, and, thus, interpretable under a specific label. Expanding upon this, both the Patient survey and Dermatology survey questionnaires included several questions of mixed format: the majority contained multiple choice questions, followed by select all questions, and lastly, one or two free response questions. The questions encompassed the nature of the treatment employed for MF and its visible effects on the skin to narrow down the most potentially efficacious treatment for MF.

The questionnaires were structured so that the questions a participant would receive were contingent upon the answer they provided to the previous question. This was part of ensuring that the participant takes the survey questionnaire under the labeled section of their respective treatment category. Furthermore, participants were asked a preliminary set of questions which asked them the type of treatment taken and how it was taken. Again, these serve to identify the type of respondent and classify them under a specific treatment category in which they would answer the remainder of the survey under. After answering the "preliminary" questions that classified participant response under the specific treatment type category, the rest of the survey was the same for each participant as they were given 
the same set of questions to follow up on under their respective treatment label. These questions can be further visualized in the Figures below.

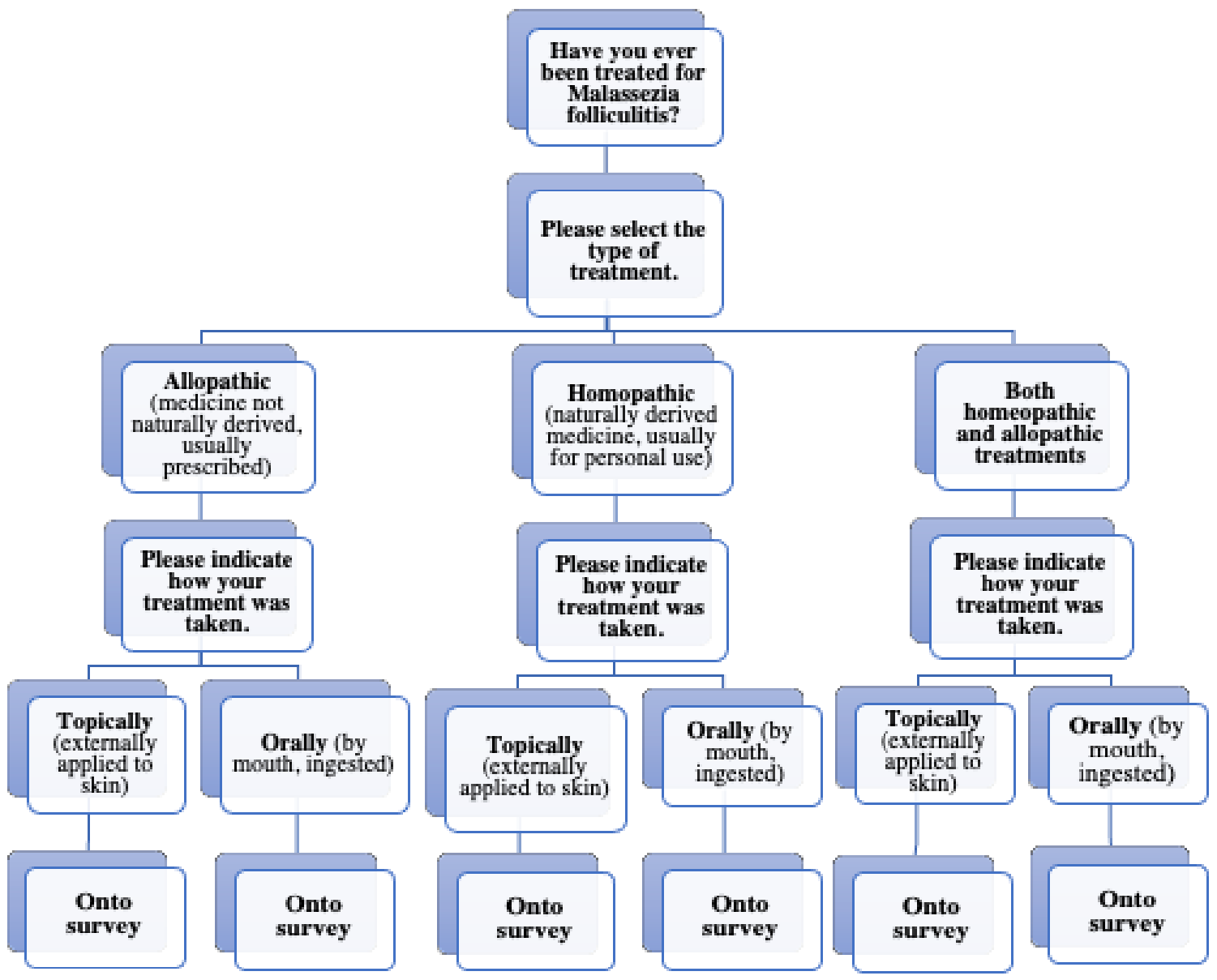

Figure 2. Patient Survey Questionnaire- Preliminary Questions. 

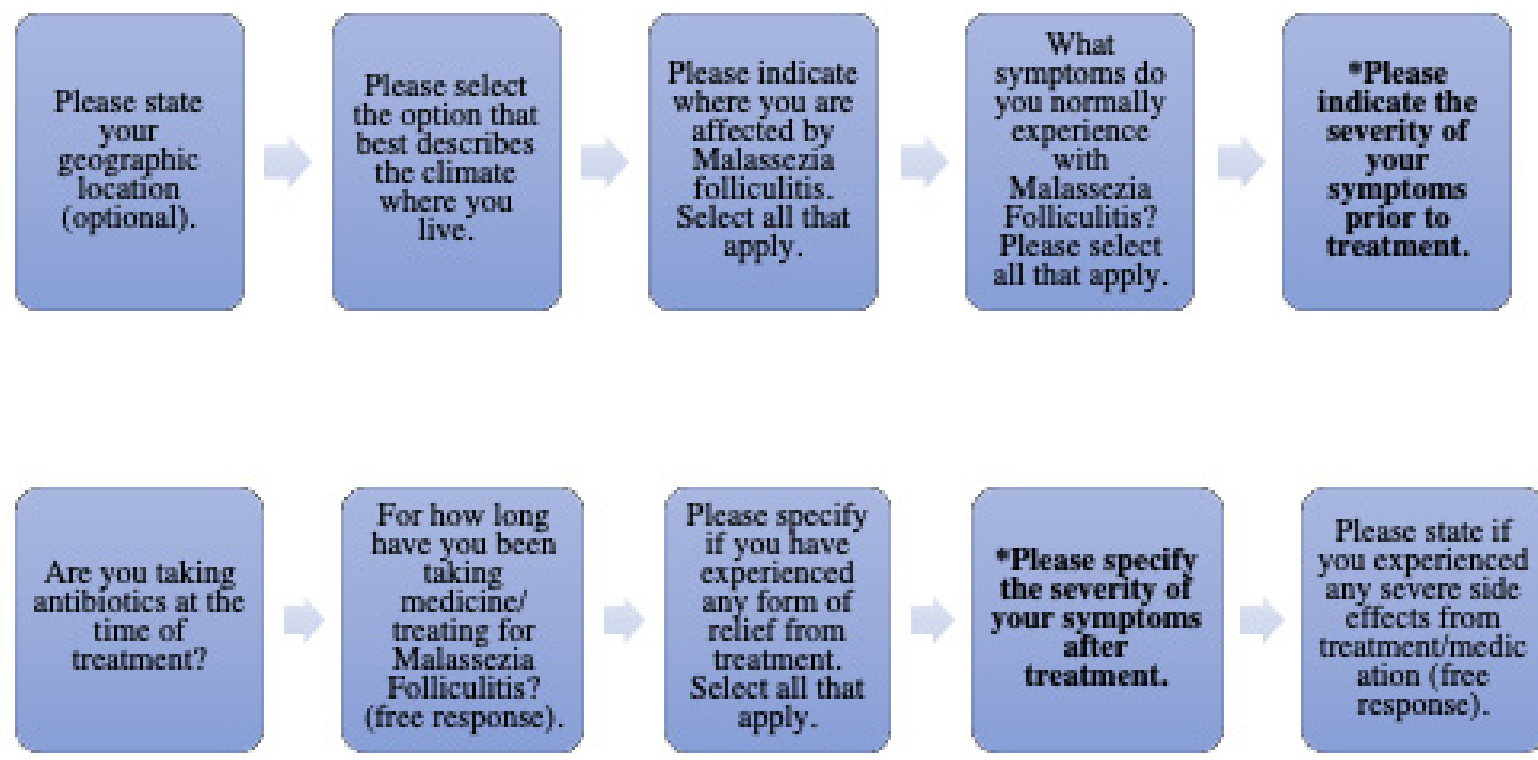

Figure 3. Patient Survey Questionnaire- Survey questions.

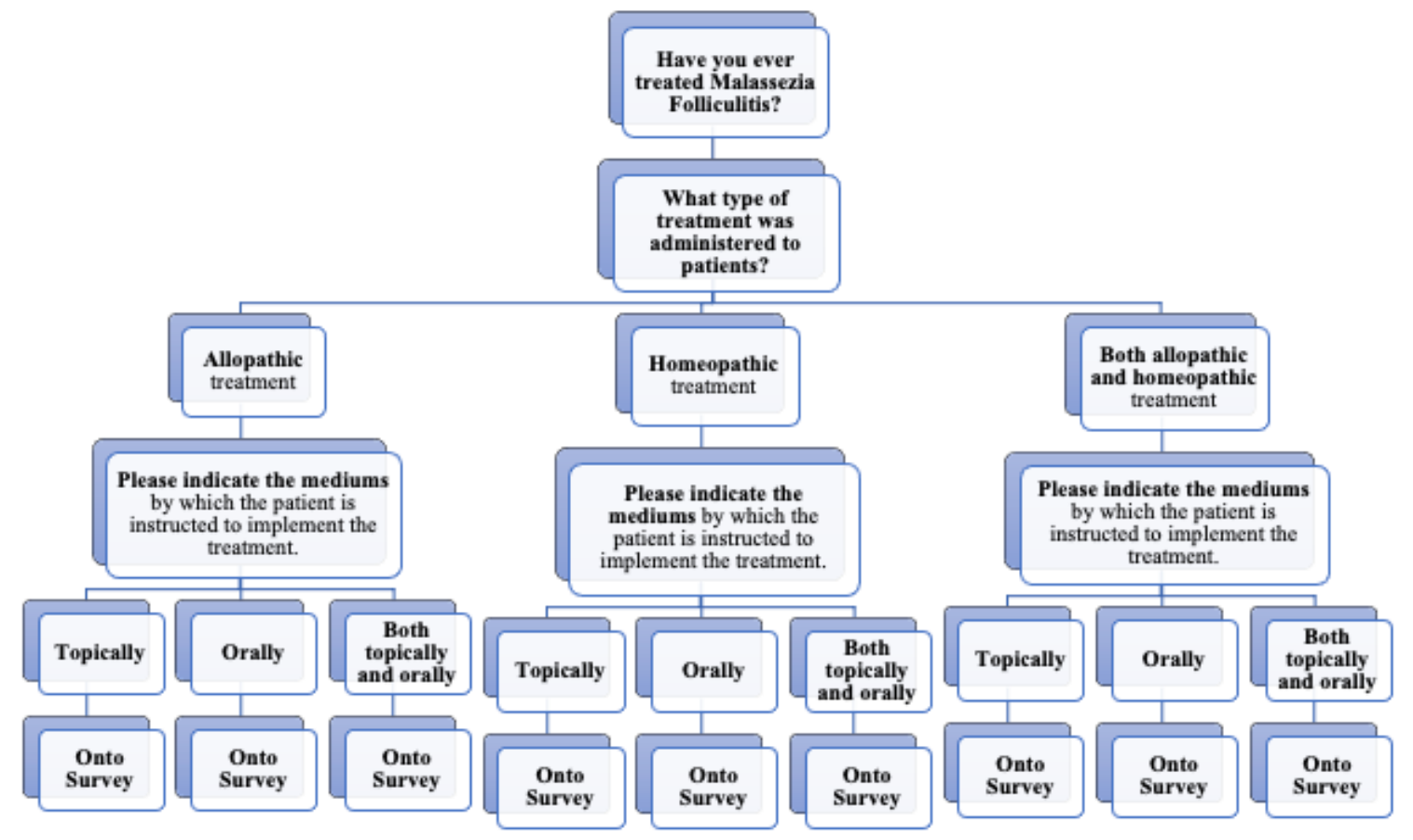

Figure 4. Dermatologist Survey Questionnaires- Preliminary Questions. 

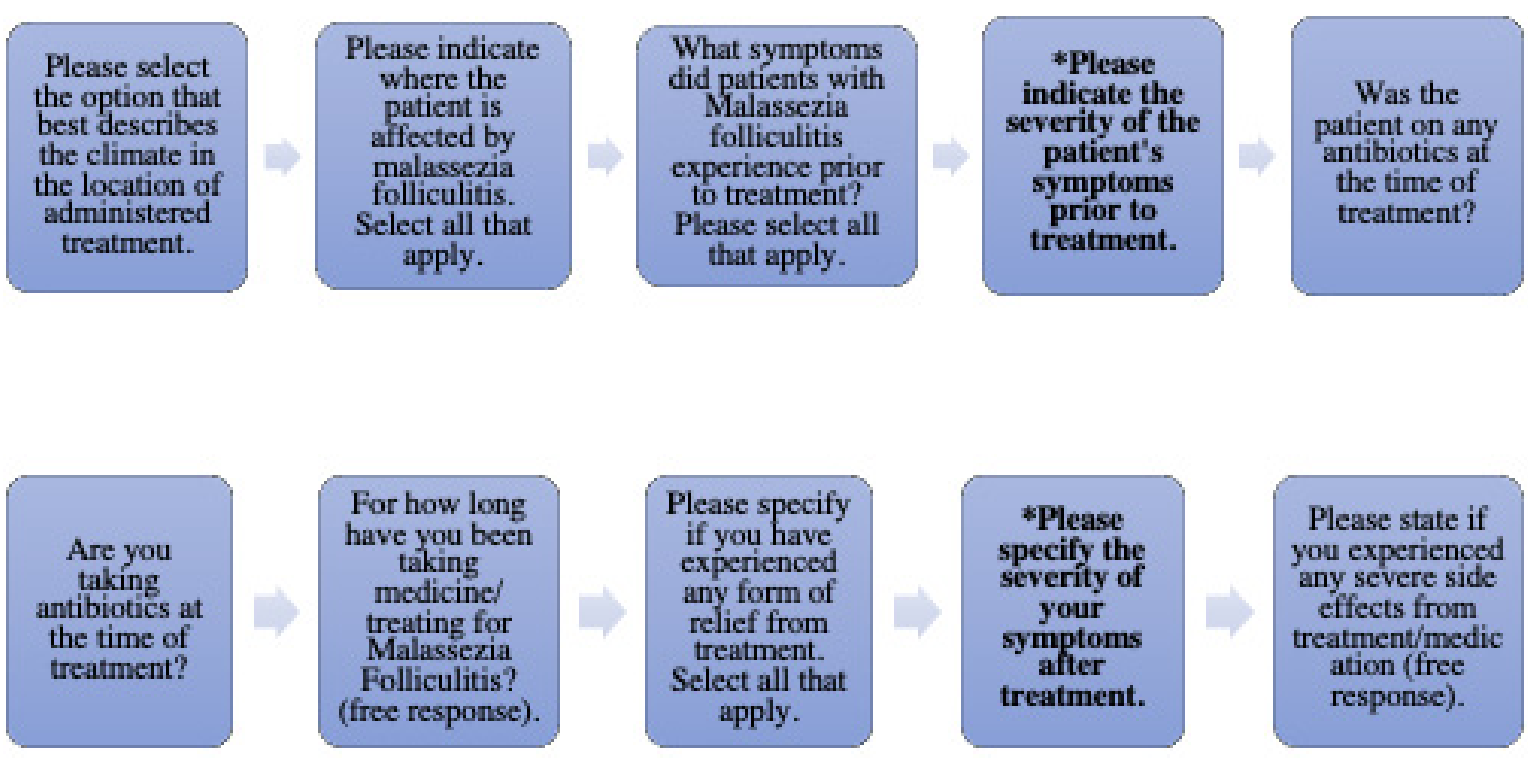

Figure 5. Dermatologist Survey Questionnaire- Survey Questions.

\section{Results}

With respect to the two variations of both distributed surveys, the results are as follows: The Patient Survey received 15 total participants, whereas the Dermatologist Survey received a total of 3 . It is important to acknowledge that the dermatologist results produced were from a total of 3 doctors, 2 of which specialized in a field: in one rheumatology and the other in dermatology, and the other remaining doctor in general medicine. Despite differences in specialization, all doctors confirmed having treated patients with MF; thus, the potential disparities of these differences are of little to no significance with regards to the validity of the results.

The Patient Survey received 16 total responses with the omission of one participant that reported having not received treatment for MF in the preliminary questions, which disqualified and removed them from participating in the survey questionnaire. Thus, the following data pertains to the 15 remaining participants who were able to respond. Again, the notations are of the following format: [Treatment type - Manner in which taken]. The participants generated data under 6 of the 9 treatment categories mentioned before: [Allopathic - Topical], [Allopathic - Oral and topical], [Homeopathic - Topical], [Homeopathic - topical and oral], a partial combination treatment [Allopathic + homeopathic - topical], and a complete combination treatment [Allopathic + homeopathic - topical + oral]. Overall, the Patient Survey received more feedback than the Dermatologist Survey.

For background, 6 out of $15(40 \%)$ of the total participants reported having been on antibiotics at the time of treatment. It is important to note that antibiotics are notoriously known for, both empirically and anecdotally, interfering with the skin's naturally occurring microbiota and encouraging the proliferation of MF [9]. 7 out of 15 (46.66\%) of the total participants were treated in continental areas, characterized by varying warm-cool summers and very cold winters, 6 out of $15(40 \%)$ in temperate areas with warm, humid summers, thunderstorms and mild winters, 1 out of $15(6.66 \%)$ in tropical (hot and humid with lots of precipitation) and 1 out of $15(6.66 \%)$ in arid areas (dry, little precipitation). Temperate and tropical climates typically have higher humidity than their drier, cooler counterparts, which is known to be optimal for MF pathogenesis and the exacerbation of its symptoms [10]. Despite these apparent setbacks, results demonstrated progress, notably for the complete combination treatment of allopathic and homeopathic medicines taken both topically and orally [Allopathic + homeopathic - topical + oral].

With respect to the physiological tendencies of MF, the data documents that 14 out of $15(93.33 \%)$ of total surveyed patients were affected on the face, 9 out of $15(60 \%)$ on the chest, 8 out of $15(53.3 \%)$ on the upper back, 6 
out of 15 (40\%) on the shoulders, and the remaining 3 (19.98\%) participants entered a custom response for "trunk," "legs and armpits," and "scalp." Prior to the administration of treatment, 12 out of 15 (80\%) total participants reported having frequently dealt with abnormalities in skin texture, 13 out of $15(86.66 \%)$ with inflammation (pimples, breakouts), and 14 out of 15 (93.33\%) with irritation (itching, redness, pain). The remaining amount was represented by one participant who reported back a custom entry for "extreme itchiness."

Thus, the data reflects that the most prominent MF- induced symptoms are irritation, inflammation, and grittiness of which have a tendency to concentrate on areas like the face and chest.

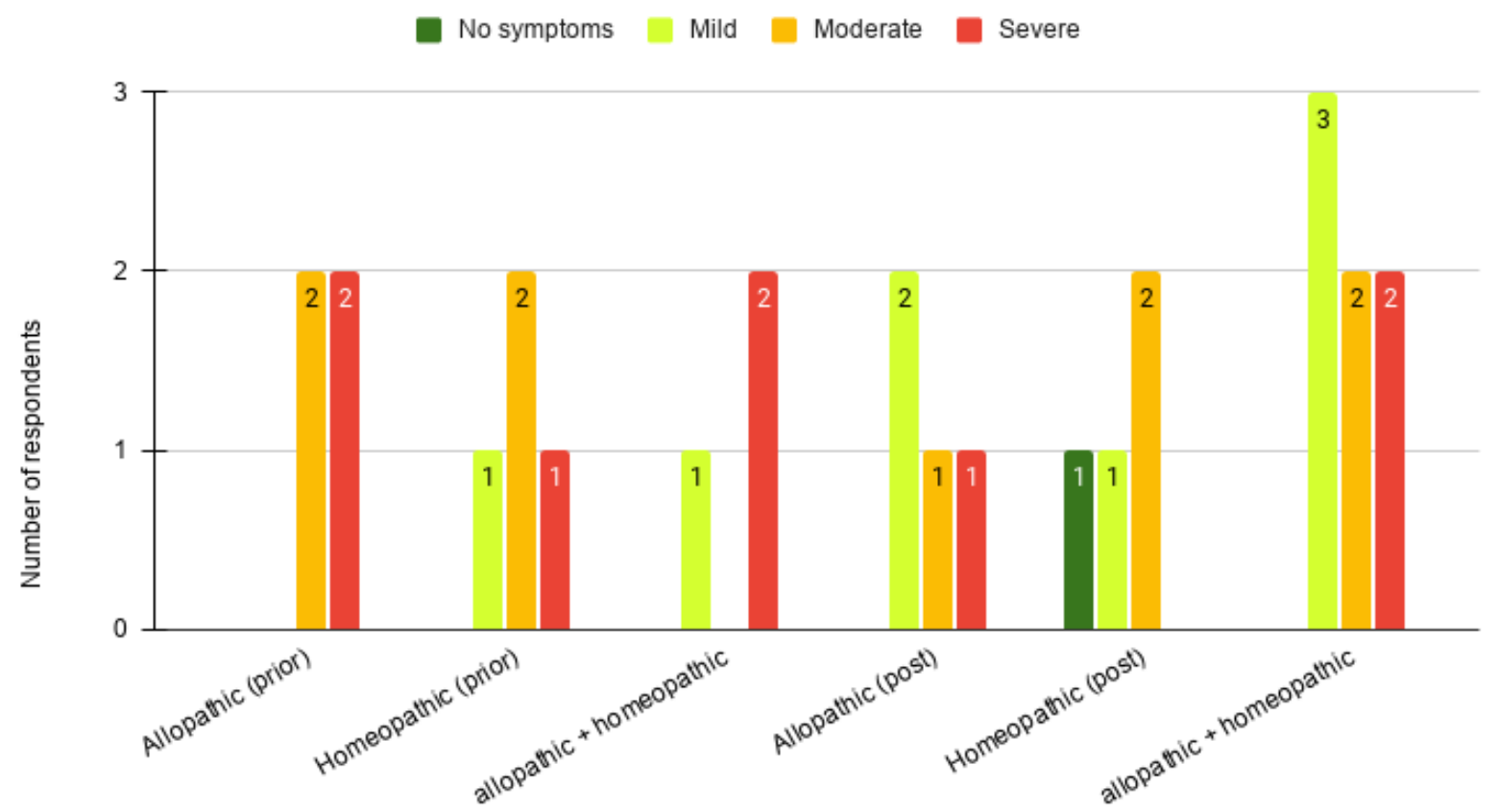

Treatment type

An abundance of patients experienced relief after treatment, with over $60 \%$ of the total participants experiencing improvement in the feel and texture of the skin, a visible reduction of inflammation and of irritation, with only $20 \%$ of participants reporting no improvement. Regarding skin severity, the data shows a significant shift in population percentage under different categories.

Prior to treatment, $50 \%$ of allopathic users experienced severe and moderate symptoms, but after treatment, that percentage has been reduced to $25 \%$. Contrastingly, homeopathic users found no change: the ratios stayed the same prior and post treatment. The data produced from the Patient Survey reveals that allopathic medicine accomplishes a more significant decrease in MF amongst patients than does homeopathic medicine. However, the data pales in comparison to the partial combination treatment [allopathic + homeopathic], which shows the greatest range difference in data. These values will be visually compared in Graph 1. On the left of the graph, which shows skin severity prior to treatment, the combination treatment shows 1 response for mild, and 2 for severe. Post treatment, however, there was an increase in reports for mild symptoms, with 3 participants reporting back the change. Moreover, 2 more participants clustered under moderate symptoms, which demonstrates improvement from the previous reports under just mild and severe reports. This suggests that a combination of allopathic and homeopathic treatments can prove more efficacious than the two alone. 
Interestingly enough, participants who had taken a combination treatment of both allopathic and homeopathic medicine had experienced a 50\% decrease in the number of participants who reported experiencing severe symptoms and a $40 \%$ increase in the number who reported experiencing mild symptoms. This means that the data used to heavily cluster under "severe" symptoms, but after combination treatment, data began to cluster under "mild" and "moderate" symptoms- largely. This suggests the efficacy of combination treatment. This suggests that although allopathic medicine (especially when taken orally) does trump homeopathic medicine alone, a combination treatment may be most efficacious in delivering the most significant results in the reduction of MF.

As a subset of the above conclusion, the Patient Survey also produced ancillary data that explored the most efficacious manner in which to take the combination treatment, represented below in Figure 7. Although it is established that a combination of allopathic and homeopathic treatments may be more efficacious than when given treatments independently, the survey data suggests that the manner in which the combination is taken can impact efficacy.

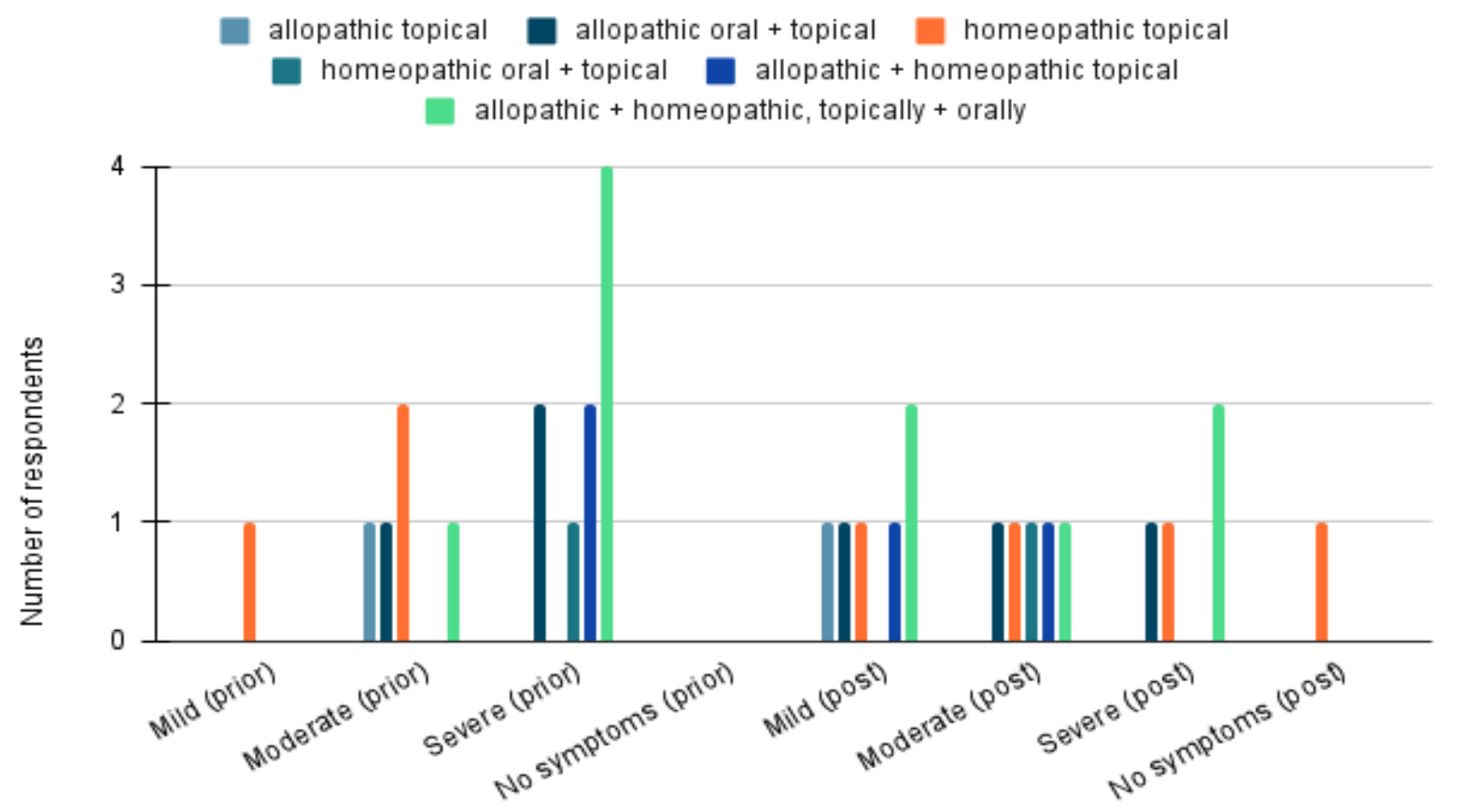

Symptom Severity

$40 \%$ of respondents reported taking antibiotics at the time of treatment. Note that one specific report from a participant said that the "antibiotics worsened it," with "it" in reference to MF. The average length of treatment was 22 days. The Dermatologist Survey showed that prior to the administration of treatment, 2 medical professionals reported that patients frequently dealt with abnormalities in skin texture as well as with inflammation and irritation, as shown below in Figure 8. Of those breakdowns, all who had been administered allopathic medicine orally had experienced inflammation and irritation prior to treatment, while all who were administered a combination of allopathic medicine both topically and orally had experienced abnormalities in skin texture, inflammation (pimples, breakouts) and irritation (itching, redness, pain) prior to treatment. The most frequently affected areas of the body were shown to be the upper back and shoulders (all doctors reported), followed by the chest ( 2 out of 3 doctors reported) and lastly, the face (1 out of 3 doctors reported), respectively. All 3 doctors reported that patients who were administered allopathic treatment reported moderate symptoms prior to treatment. In the follow-up visits, the medical professionals noted an egregious improvement in the skin of their patients, with a complete eradication of MF for those who were administered a 
combination of allopathic medicine both topically and orally; whereas those who administered solely allopathic medicine orally, without combination, saw an incomplete, partial eradication. This shows that 2 out of 3 doctors who used a combination treatment found it to be completely successful, while those who did not employ a combination treatment, despite using allopathic medicine, did not encounter a complete success. The relief was characterized by an improvement in the feel and texture of the skin, a visible reduction of inflammation, and a reduction of irritation. More specifically, patients who were administered the combination of allopathic medicine topically and orally [allopathic topical + oral] experienced a complete relief with all of their previous skin problems.

Furthermore, patients who were administered solely allopathic medicine orally [allopathic - oral] reduced their overall skin severity from "moderate" to "mild," whereas those who were administered the combination of allopathic medicine both topically and orally [allopathic - topical + oral] reduced their overall skin severity from "moderate" to "no symptoms." Thus, the data demonstrates a greater leap of improvement in skin severity in patients who were administered allopathic medicine both topically and orally than just orally alone, suggesting the greater efficacy of a combination treatment in eradicating MF. When compared with the data from the Patient Survey, the argument for the efficacy and versatility of a combination treatment for MF remains strongly evident, and specifically one that encompasses all combinations of the medicine types: An allopathic and homeopathic dosage used both topically and orally [allopathic + homeopathic - topical + oral]. To note, none of the total patient population experienced any severe side effects from treatment, and the average length of treatment was 22 days.

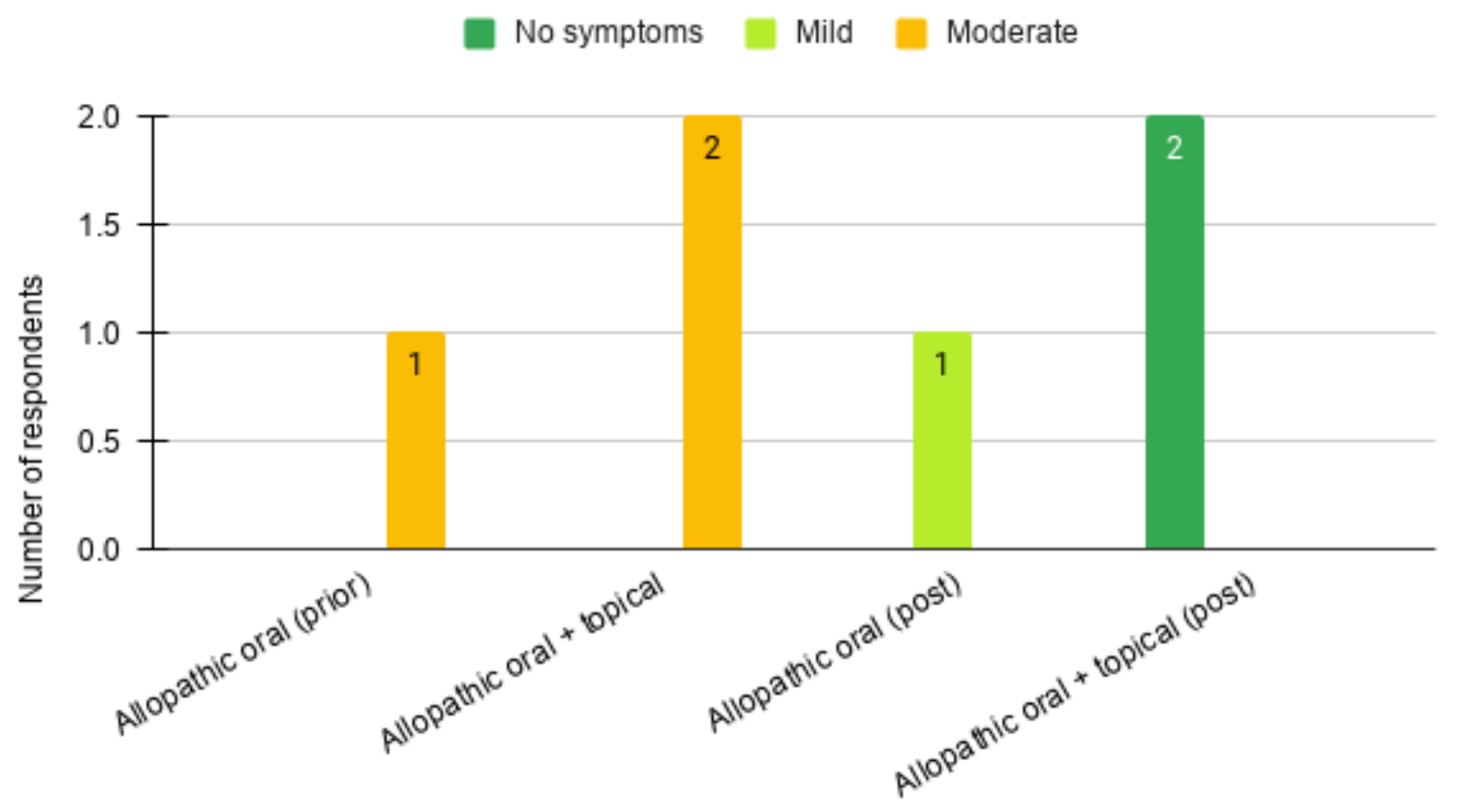

Treatment type

Discussion

Given that across both survey questionnaires, allopathic medicine taken orally [allopathic - oral] and homeopathic medicine applied topically [homeopathic - topical] delivered the most significant individual improvement, the data suggests that a combination administration: allopathic and homeopathic medicine taken in both topical and oral form [allopathic + homeopathic - topical + oral], is most promising in reducing MF. This is further exemplified through the $66 \%$ decrease in the number of participants who reported experiencing severe symptoms and the $42 \%$ increase in the number who experienced mild symptoms. These statistics are similar to the ones of the simple combination 
treatment of allopathic and homeopathic medicine mentioned previously, though they are slightly higher, indicating more improvement. This rationalizes why addressing the manner in which medicine is taken is significant to the eradication of MF.

As all of its responses collected data only for "allopathic" treatment, the Dermatologist Survey produces no statistical standard of comparison independently, which renders it infeasible to have any independent conclusions drawn from it regarding the comparative efficacies of allopathic and homeopathic treatment types. To reconcile these disparities, the Dermatologist Survey was compared to the Patient Survey to identify underlying trends in data amongst both patient and medical professional populations and concluded that allopathic treatments often work better than homeopathic ones. It found further, however, that a complete combination treatment of allopathic and homeopathic medication taken topically and orally [allopathic + homeopathic - topical + oral] was most efficacious in reducing dermal Malassezia folliculitis. Such is represented by Table 1, below.

Table 1. Overlapping Data Trends between the Patient Survey and Dermatologist Survey.

\begin{tabular}{|c|c|c|c|}
\hline & Patient Survey Results & Both & $\begin{array}{l}\text { Dermatologist Survey Re- } \\
\text { sults }\end{array}$ \\
\hline $\begin{array}{l}\text { Allopathic treatment as } \\
\text { a whole (taken either } \\
\text { topically or orally) }\end{array}$ & $\begin{array}{l}\text { From severe symptoms } \\
\text { prior to treatment to } \\
\text { mostly mild and moderate } \\
\text { post treatment. }\end{array}$ & & $\begin{array}{l}\text { From all experiencing mod- } \\
\text { erate severity prior to treat- } \\
\text { ment, to mild and no symp- } \\
\text { toms post treatment. }\end{array}$ \\
\hline Allopathic oral + topical & $\begin{array}{l}\text { From all experiencing se- } \\
\text { vere symptoms prior to } \\
\text { treatment to an even distri- } \\
\text { bution. of mild, moderate, } \\
\text { and severe post treatment. }\end{array}$ & $\begin{array}{l}\text { Significant improvement. } \\
\text { Efficacious treatment. }\end{array}$ & $\begin{array}{l}\text { All users saw a full eradica- } \\
\text { tion of MF. From moderate } \\
\text { symptoms prior to treat- } \\
\text { ment to no symptoms post } \\
\text { treatment. }\end{array}$ \\
\hline Allopathic oral alone & & & $\begin{array}{l}\text { Incomplete but significant } \\
\text { eradication of MF. From } \\
\text { moderate symptoms to } \\
\text { mild. }\end{array}$ \\
\hline
\end{tabular}

\section{Conclusion and Implications}

Evidently, the Dermatologist Survey reaffirms the efficacy of allopathic medicine over homeopathic medicine (holistically and generally, taken either orally or topically), as was demonstrated in the findings of the Patient Survey. The overlaps in the data also further the principle of using a combination treatment for the strongest eradication of MF, which is implementing allopathic and homeopathic treatment both topically and orally. Regarding the purpose of the research, the conclusion is that allopathic treatment is in fact wholly more effective than homeopathic treatment when compared independently. The study produced a further finding that closed unfinished hypotheses in previous dermatological studies that proposed that a combination treatment was most effective. The final finding from the study was that which further described the most efficacious form in which to take treatment, which is both topically and orally. The data holistically suggested that the combination treatment, [allopathic + homeopathic - topical + oral] could be most efficacious in eradicating MF. 


\section{Future Research}

As the study suggested that homeopathic medicines do, in fact, have merit in the medical sphere, the usage of these medicines should be further researched in a setting that controls confounders such as diet and climate. The efficacious potentiality of the combination treatment [allopathic + homeopathic - topical + oral] across different regions should also be studied. Doing this will provide more clarity on the individual efficacy of the treatment unaffected by potential confounders, and can assist in reaching a more definite, thorough conclusion on the comparative efficacies of allopathic vs. homeopathic treatments in reducing MF. The aim of the study is then to encourage dermatologists and doctors to focus attention on and consider combination treatments that include homeopathic medicine as an improver of $\mathrm{MF}$, since the inclusion of homeopathic medicines as part of combination treatments has proved to hold promise. In considering this, more research can be done in the dermatological sphere to reconcile the aforementioned limiting disparities of this study: climate, diet, COVID-19. The overarching aim is that, with more research, more attention is given to the potential relief and satisfaction of patients and dermatologists alike with respect to $\mathrm{MF}$.

\section{Limitations}

Recall that in the Patient Survey, $40 \%$ of respondents reported taking antibiotics at the time of treatment. Antibiotics, as mentioned previously, can interfere with the skin's microbiome and tamper with MF growth. This could affect data relating to treatment efficacy, though, in this study, no significant impact was found.

The Dermatologist Survey received especially less participants than the Patient Survey which was due largely in part to COVID-19 and its restrictions on social activity. As it was holistically difficult to get responses from dermatology offices, the Dermatologist Survey results produced came from a total of 3 doctors, 2 of which specialized in a field: in one rheumatology, one in general medicine, and the other in dermatology. Furthermore, patients were in a tropical environment at the time of treatment, which, again, is characterized by high levels of precipitation and humidity known to be optimal for MF pathogenesis and the exacerbation of its symptoms [10]. This setback did not seem to be of much significance to the data in this survey, however, as different outcomes still persisted.

\section{Acknowledgements}

I would like to express my profound gratitude for those who have aided my research process. I want to give thorough thanks my teacher Mr. Aaronson, who has meticulously monitored and guided every step of my research. Next, I want to thank Ms. Ramkissoon, who opened my eyes to scientifically sound approaches in my research. I want to thank my friends and peers who were always eager to give constructive criticism and cheer me on, and also the doctors that took their time to participate in my surveys, especially during trying times like the pandemic we are witnessing. Lastly, I want to vehemently express my utmost gratitude to my parents who always encouraged and supported me, every step of the way.

\section{References}

[1] Hald, M., Arendrup, M. C., Svejgaard, E. L., Lindskov, R., Foged, E. K., Saunte, D. M., \& Danish Society of Dermatology (2015). Evidence-based Danish guidelines for the treatment of Malassezia related skin diseases. Acta dermato-venereologica, 95(1), 12-19. https://doi.org/10.2340/00015555-1825

[2] Durdu, M., Güran, M., \& Ilkit, M. (2013). Epidemiological characteristics of Malassezia folliculitis and use of the May-Grünwald-Giemsa stain to diagnose the infection. Diagnostic microbiology and infectious disease, 76(4), 450-457. https://doi.org/10.1016/j.diagmicrobio.2013.04.011 
[3] Bahlou, E., Abderrahmen, M., Fatma, F., Sellami, K., Sonia, B., Mseddi, M., Amouri, M., Turki, H., Fatma, C., \& Ayedi, A. (2018). Malassezia Folliculitis: Prevalence, Clinical Features Risk Factors and Treatment: A Prospective Randomized Comparative Study. http://www.imedpub.com/journal-immunology-microbiologyl

[4] Ayers, K., Sweeney, S. M., \& Wiss, K. (2005). Pityrosporum folliculitis: diagnosis and management in 6 female adolescents with acne vulgaris. Archives of pediatrics \& adolescent medicine, 159(1), 64-67. https://doi.org/10.1001/archpedi.159.1.64

[5] Donato, R., Sacco, C., Pini, G., \& Bilia, A. R. (2020). Antifungal activity of different essential oils against Malassezia pathogenic species. Journal of ethnopharmacology, 249, 112376. https://doi.org/10.1016/j.jep.2019.112376

[6] Vlachos, C., Henning, M., Gaitanis, G., Faergemann, J., \& Saunte, D. M. (2020). Critical synthesis of available data in Malassezia folliculitis and a systematic review of treatments. Journal of the European Academy of Dermatology and Venereology:JEADV, 34(8), 1672-1683. https://doi.org/10.1111/jdv.16253

[7] Theelen, B., Cafarchia, C., Gaitanis, G., Bassukas, I. D., Boekhout, T., \& Dawson, T. L., Jr (2018). Malassezia ecology, pathophysiology, and treatment. Medical mycology, 56(suppl_1), S10-S25. https://doi.org/10.1093/mmy/myx134

[8] Gupta, A. K., Batra, R., Bluhm, R., Boekhout, T., \& Dawson, T. L., Jr (2004). Skin diseases associated with Malassezia species. Journal of the American Academy of Dermatology, 51(5), 785-798. https://doi.org/10.1016/j.jaad.2003.12.034

[9] Rubenstein, R. M., \& Malerich, S. A. (2014). Malassezia (pityrosporum) folliculitis. The Journal of clinical and aesthetic dermatology, 7(3), 37-41. https://www.ncbi.nlm.nih.gov/pmc/articles/PMC3970831/

[10] Kim SY, Lee YW, Choe YB, Ahn KJ. Progress in Malassezia Research in Korea. Ann Dermatol. 2015;27(6):647-657. doi:10.5021/ad.2015.27.6.647 